\title{
Silicon and tungsten oxide nanostructures for water splitting
}

Karla R. Reyes Gil, Joshua M. Spurgeon, Nathan S. Lewis

Karla R. Reyes Gil, Joshua M. Spurgeon, Nathan S. Lewis, "Silicon and tungsten oxide nanostructures for water splitting," Proc. SPIE 7408, Solar Hydrogen and Nanotechnology IV, 74080 S (20 August 2009); doi: 10.1117/12.825545

SPIE Event: SPIE Solar Energy + Technology, 2009, San Diego, California, United States 


\title{
Silicon and tungsten oxide nanostructures for water splitting
}

\author{
Karla R. Reyes Gil", Joshua M. Spurgeon, Nathan S. Lewis \\ California Institute of Technology, 1200 E California Blvd, Pasadena, CA, USA 91125
}

\begin{abstract}
Inorganic semiconductors are promising materials for driving photoelectrochemical water-splitting reactions. However, there is not a single semiconductor material that can sustain the unassisted splitting of water into $\mathrm{H}_{2}$ and $\mathrm{O}_{2}$. Instead, we are developing a three part cell design where individual catalysts for water reduction and oxidation will be attached to the ends of a membrane. The job of splitting water is therefore divided into separate reduction and oxidation reactions, and each catalyst can be optimized independently for a single reaction. Silicon might be suitable to drive the water reduction. Inexpensive highly ordered $\mathrm{Si}$ wire arrays were grown on a single crystal wafer and transferred into a transparent, flexible polymer matrix. In this array, light would be absorbed along the longer axial dimension while the resulting electrons or holes would be collected along the much shorter radial dimension in a massively parallel array resembling carpet fibers on a microscale, hence the term "solar carpet". Tungsten oxide is a good candidate to drive the water oxidation. Self-organized porous tungsten oxide was successfully synthesized on the tungsten foil by anodization. This sponge-like structure absorbs light efficiently due to its high surface area; hence we called it "solar sponge".
\end{abstract}

Keywords: water splitting, silicon, tungsten oxide, solar cell, membrane, hydrogen production, semiconductor

\section{INTRODUCTION}

\subsection{Hydrogen Production}

An increased awareness of environmental issues and limited oil reserves has led to a new focus on the development of clean and renewable energy sources. Hydrogen holds the potential to provide clean, safe, affordable, and secure energy from abundant domestic resources. Using hydrogen as a form of energy can not only reduce our dependence on imported oil, but also benefit the environment by reducing emissions of greenhouse gases and criteria pollutants that affect our air quality. Hydrogen is a promising energy carrier (like electricity) that can be used to power nearly every end-use energy need. It stores and delivers energy in a usable form, but it must be produced from compounds that contain it (water, methane and biomass). Hydrogen is versatile in part because it can be produced from different and abundant energy sources, including fossil (such as coal and natural gas), nuclear, and renewable (wind, solar, geothermal, and hydroelectric power).

The popular perception is that hydrogen is always "green". However, hydrogen is only as clean or dirty as the manufactured process used to produce it. ${ }^{1}$ According with the Department of Energy, most of the hydrogen produced today in the United States (95\%) is made via steam methane reforming. Although natural gas reforming is relatively mature compared to other hydrogen production technologies, the capital equipment, operation, and maintenance costs associated with distributed natural gas reforming must be reduced to make hydrogen cost-competitive with the conventional fuels we use today. In addition, natural gas resources in the United States are limited, and this producing large amount of hydrogen from natural gas is unsustainable for the long term, as it would essentially trade U.S. dependence on imported oil for U.S. dependence on imported natural gas. However, the most important drawback of this technique is the $\mathrm{CO}_{2}$ emissions. As the result, new and efficient carbon sequestration techniques are needed to avoid the $\mathrm{CO}_{2}$ emissions.

*E-mail: kreyes@caltech.edu; phone: 1626 395-2712

Solar Hydrogen and Nanotechnology IV, edited by Frank E. Osterloh, Proc. of SPIE Vol. 7408, 74080 S - (c) 2009 SPIE · CCC code: 0277-786X/09/\$18 - doi: 10.1117/12.825545 
For a completely carbon-free hydrogen production, fossil sources have to be replaced for nuclear power and/or renewable carbon-neutral energy sources. To depend only in nuclear power to supply all the energy required for hydrogen production, an enormous number of nuclear reactors are needed. According with Nathan S. Lewis, we need to build a new nuclear power plant every other day, continuously, for the next 50 straight years to meet the world energy needs. $^{2}$ Some of the general concerns about this massive reactor construction are the limited uranium supply in the earth, nuclear waste handling and community opposition.

Some renewable sources, such as wind, and geothermal, are uneven distributed in the world and cannot be used as a global scale. These local sources are limited to some geographic locations and could initiate even more political and military conflicts. Solar energy appears to be the renewable source with the greatest potential. However, the production of hydrogen from renewable sources is still very expensive in comparison with the energy from fossil fuels. In general, research is focused on reducing production costs by improving the efficiency of hydrogen production technologies. The greatest challenge to hydrogen production is to become cost-competitive with conventional fuels.

\subsection{Solar water splitting}

Photoelectrochemical (PEC) systems produce hydrogen from water using sunlight and photo-electrochemical materials (specialized semiconductors) that absorb sunlight and use the light energy to dissociate water molecules into hydrogen and oxygen. ${ }^{3}$ This technology offers important advantages in comparison to other hydrogen production technologies. First, this fuel is completely clean during the production and combustion of hydrogen. Second, solar power is universally available, while other renewable sources, such as wind, tidal, geothermal, hydroelectric, and hydrothermal power, are more regionally based. Third, solar power is enormous. The sun provides Earth with 120,000 TW, meaning that more energy from the sun hits the earth in one hour than all of the energy consumed on our planet in an entire year. Solar energy is the only renewable source with the potential to satisfy alone the carbon-free energy supply required for current and future generations. Figure 1 shows the actual land area required for a solar farm to match the energy currently consumed in our country. ${ }^{2}$

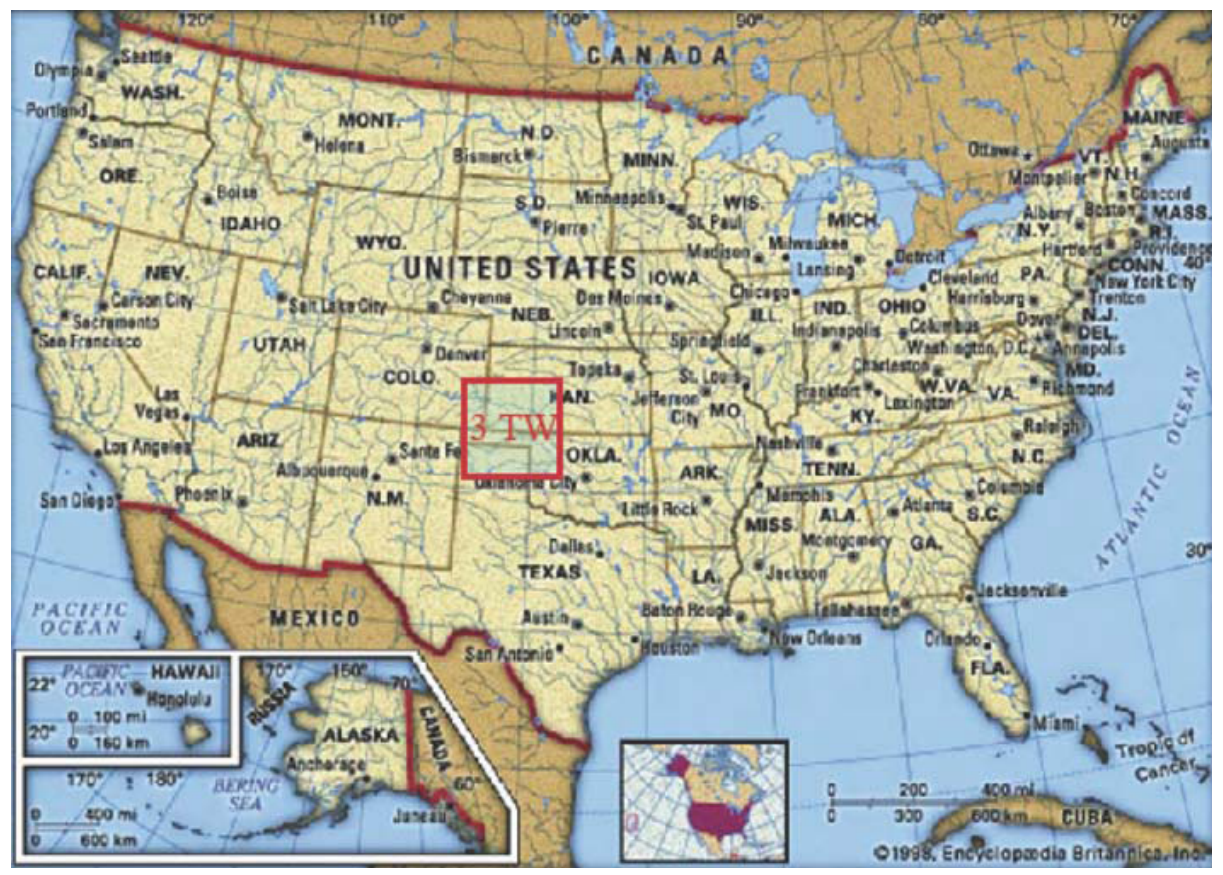

Figure 1. The red square shows the amount of land needed for a solar farm operating at $10 \%$ efficiency to match the $3 \mathrm{TW}$ of power currently consumed in the United States. ${ }^{2}$ 


\subsection{Statement of the problem}

For commercial use, the photoelectrochemical water splitting cell must have an efficiency of 7-10\%, a life-time of 10 years and low fabrication and maintenance costs. The overall goal is to develop an efficient, stable and cost effective semiconductor material that will split water using sunlight as the only energy input. However, the principal problem in the solar-to-hydrogen conversion field is that there is not a single material that is efficient for overall water splitting, as well as durable, non-toxic and non-expensive.

\section{THREE-COMPONENT SOLAR WATER SPLITTING CELL}

\subsection{Previous solar cell design}

The simplest solar-derived water splitting system imaginable would consist of a suspension of photocatalytic particles in water. The particles would absorb sunlight and then directly create chemical fuels as $\mathrm{H}_{2}$ and $\mathrm{O}_{2}$ gases, with no need for production of electrical power as an intermediary. This approach would avoid the balance of systems costs associated with discrete production and consumption of electrical energy to then be fed into a separate electrolyzer, and would additionally offer the opportunity for development of truly inexpensive solar energy absorption and conversion systems. However, this water splitting system has several drawbacks. First, the hydrogen and oxygen produced in this system will need to be separated downstream, as they will be produced spatially very close to each other. Another problem is the recombination of the hydrogen and oxygen in periods of weak illumination or darkness. Finally, the inherent explosion tendency will also be serious problem with such a system design.

\subsection{Overview of the three-component solar water splitting device}

A preferred method to avoid generation of hydrogen and oxygen in the same physical space at the same time is therefore to support the photocatalyst assembly in a membrane. The hydrogen and oxygen can then be produced on the different sides of the membrane by controlling the charge separation such that electrons are shuttled to one side of the membrane and holes to the other. In this type of system, the membrane will have to be ionically conducting to allow charge balance to be maintained on both sides of the membrane. This approach closely resembles the well-established technology of a membrane-electrode-assembly of a fuel cell, except that the input energy for photoelectrolysis is provided by sunlight (Figure 2).

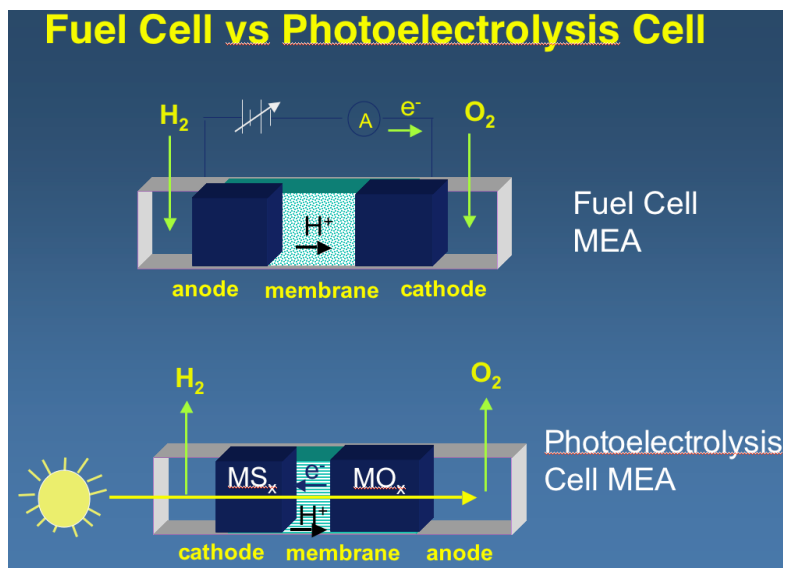

Figure 2. Schematic of similarities and differences between a $\mathrm{H}_{2} / \mathrm{O}_{2}$ Fuel Cell and the Membrane-Supported Photocatalytic Formation of $\mathrm{H}_{2}$ and $\mathrm{O}_{2}$ by Water Splitting. 
The Lewis group has been working on the development of a three-component solar water splitting system (Figure 3). The components are: (I) a membrane-supported assembly that captures sunlight and efficiently creates separated electrons and holes having sufficient chemical potential to drive the water-splitting reactions; (II) a two-electron catalyst to facilitate reduction of water to $\mathrm{H}_{2}$ at the cathode; and (III) a four-electron catalyst for water oxidation to $\mathrm{O}_{2}$ at the anode. This system design which utilizes both a photoanode and a photocathode; the job of splitting water is therefore divided into separate reduction and oxidation reactions, each of which can be optimized independently.

The polymeric membrane layer between the two electrodes must fulfill a crucial function in order for this water-splitting device to succeed. In addition to providing structural support to the rod assemblies and separation of the $\mathrm{H}_{2}(\mathrm{~g})$ and $\mathrm{O}_{2}(\mathrm{~g})$ products, the membrane will have to provide a conduction path for electrons between the photoanode and photocathode, while simultaneously acting as an exchange-membrane for the hydroxide anions (in alkaline media) or protons (in acidic media). The membrane layer must also be optically transparent, to ensure that the incident light effectively reaches both arrays of semiconductor structures. Currently, member of Lewis group are working with the development of this membrane and the solar water cell device assembly. However, the scope of this paper is the discussion of the progress on the development of the semiconductor materials that will be used as the photocathode and the photoanode.

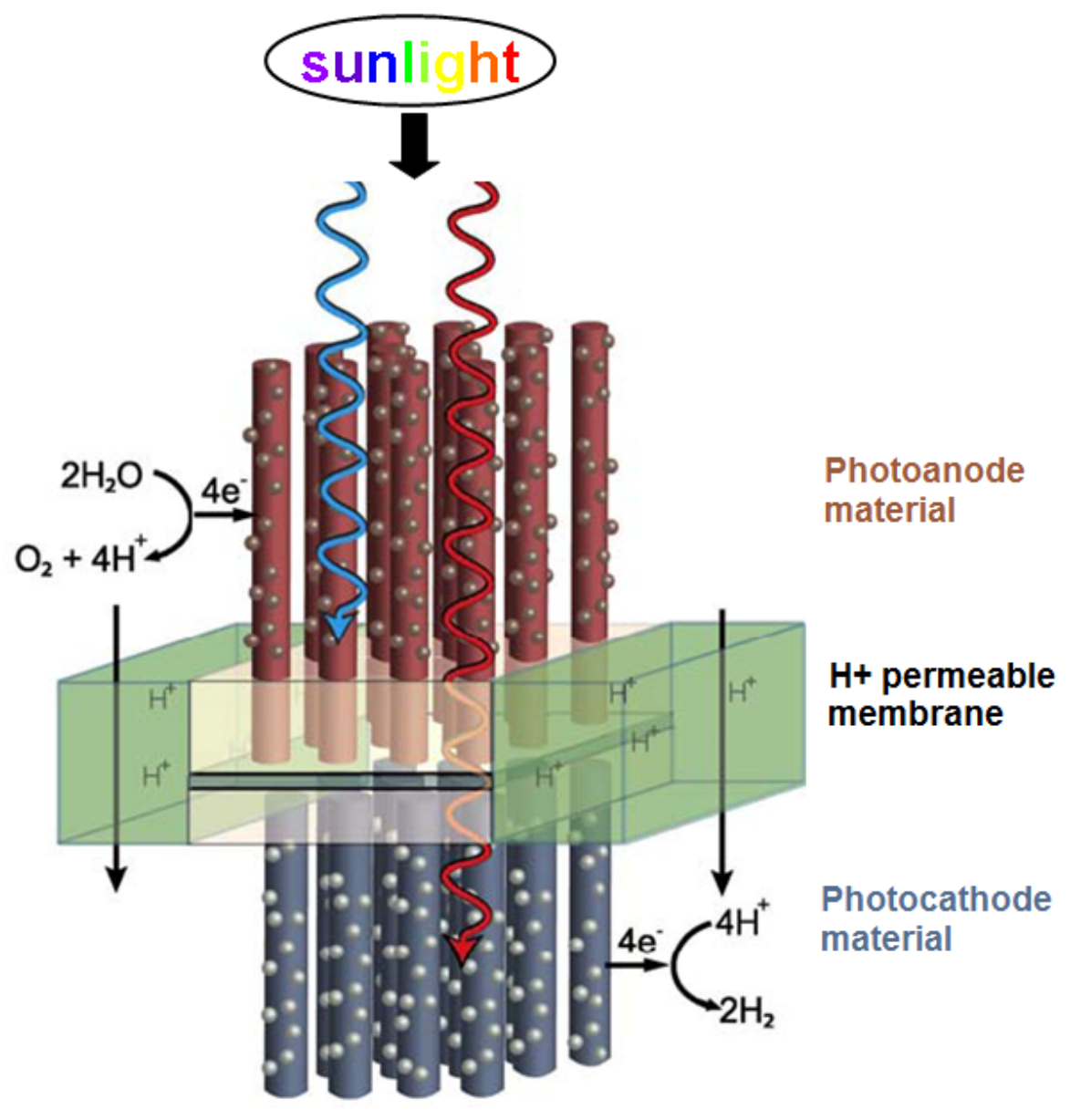

Figure 3. Schematic of the proposed solar powered water-splitting device incorporating two separate semiconductor rodarray photoelectrodes that sandwich an electronically and ionically membrane.

As mentioned in the introduction, the overall goal is the developing of efficient, durable and cost effective semiconductors for solar water splitting. The semiconductor materials must provide the necessary voltage ( $1.23 \mathrm{~V}$ under standard conditions at $1 \mathrm{~atm}$ of pressure) needed to thermodynamically split water into hydrogen and oxygen. In addition, 
the semiconductor source should be inexpensive as well as its manufacture. This requirement eliminates the use of large area single crystals. Finally, the materials must be in a configuration that allows such relatively inexpensive materials to meet a minimum efficiency threshold (around 7-10\%) so that the $\mathrm{H}_{2}$ could be produced in a cost-competitive fashion with that obtained from fossil sources at the present time. To date, there is not a single material that fulfills all the cost, durability and efficiency requirements. On the other hand, using two photoelectrodes allows for greater flexibility in materials and design, rather than relying on finding the one ideal semiconductor as the solar absorber.

The optimal band gap for a cell using a single semiconductor is between 2.0 and $2.6 \mathrm{eV}$ (considering that the bad gap overlaps the electrochemical potential needed to split water). ${ }^{4,5}$ Under solar illumination (Air Mass 1.5 solar spectrum at $100 \mathrm{~mW} \mathrm{~cm}$ ), a semiconductor with a bandgap of $2 \mathrm{eV}$ could absorb approximately $9.1 \times 10^{16}$ photons.cm ${ }^{-2} \cdot \mathrm{s}^{-1}$. In optimal conditions, each absorbed photon produces an electron-hole pair, resulting in a photocurrent density of $9.1 \times 10^{16}$ photons. $\mathrm{cm}^{-2} . \mathrm{s}^{-1}$ or $14.6 \mathrm{~mA} \mathrm{~cm}{ }^{-2}$. Using the limiting assumption that each mole of electron-hole pairs creates half a mole of $\mathrm{H}_{2}$ and $1 / 4$ mole of $\mathrm{O}_{2}, 7.6 \times 10^{-8}$ moles of $\mathrm{H}_{2} \cdot \mathrm{cm}^{-2} \mathrm{~s}^{-1}$ would be produced. The maximum efficiency that could therefore be obtained is $18 \%\left[1.23 \mathrm{eV}\right.$ (the stored free energy in the $\mathrm{H}_{2}$ and $\mathrm{O}_{2}$ formed) multiplied by $\left.14 \mathrm{~mA} \mathrm{~cm}{ }^{-2}\right]$. If the band gap of the material is $2.6 \mathrm{eV}$, the limiting efficiency is approximately $10 \%$. In our three-component cell design, the band gap of both photoanode and photocathode can be better matched to the solar spectrum (1.1 to $1.4 \mathrm{eV})$. A 1.2 $\mathrm{eV}$ bandgap material could absorb $2.5 \times 10^{17}$ photons $\mathrm{cm}^{-2} \mathrm{~s}^{-1}$; since there are two absorbers in this case, $1.25 \times 10^{17}$ electron-hole pairs $\mathrm{cm}^{-2} \mathrm{~s}^{-1}$ would be generated and would be used for splitting water into $10^{-7}$ moles $\mathrm{H}_{2} \mathrm{~cm}^{-2} \mathrm{~s}^{-1}$, therefore producing a limiting efficiency of over $24 \%$. The limiting hydrogen production efficiency using two nearly optimal band gap electrodes will therefore be greater than that available using one large band gap material.

\subsection{Silicon as a photocathode material}

Silicon, an abundant semiconductor that effectively absorbs the solar spectrum, might be suitable to drive the cathodic (hydrogen producing) component of the overall electrochemical reaction. Unfortunately, silicon is expensive in highpurity wafer formats. This section will report progress in the Lewis group on the development of p-silicon micro-rod photocathode arrays grown from inexpensive silicon sources. ${ }^{7-9}$

\subsubsection{Rod Array Geometry}

In traditional planar photovoltaic or photoelectrochemical junctions, the directions of light absorption and of chargecarrier collection are mutually parallel. Charge carriers generated deep within the bulk must necessarily traverse the full thickness of the absorbing semiconductor material in order to be collected. In the case of a planar junction solar cell, a photogenerated electron or hole created deep within the material must be able to travel all the way to the junction without recombining so that it can be collected to produce current. Accordingly, the requisite long minority-carrier diffusion length, L, explicitly demands high material purity to minimize bulk carrier recombination and implicitly requires significant material preparation costs. 

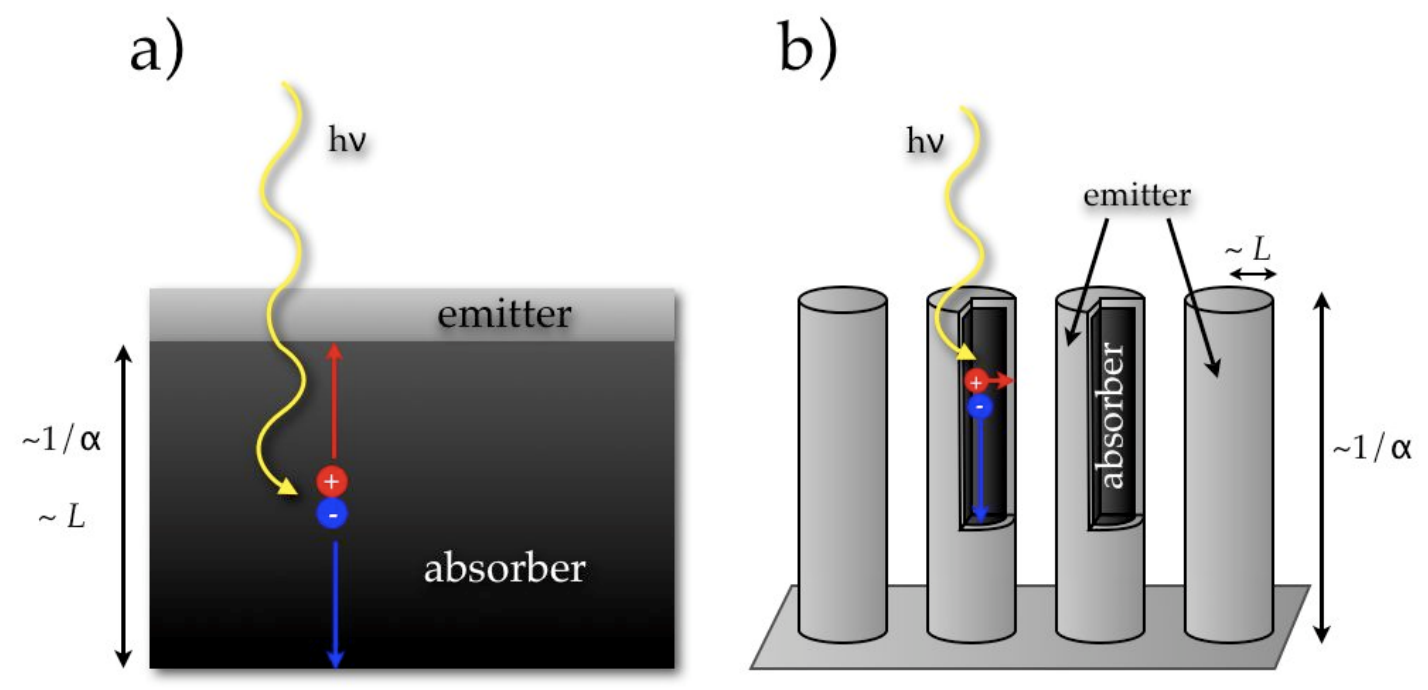

Figure 4. Comparison of the light absorption pathlength depth $(1 / \alpha)$ and the minority charge-carrier collection length, $L$, in semiconductor photovoltaic or photoelectrochemical junctions in (a) planar and (b) rod geometries.

Radial junction rod array solar cells, on the other hand, have the potential to decouple the directions of light absorption and charge carrier collection so that a semiconductor with a carrier diffusion length shorter than its absorption depth (i.e. - a lower quality, cheaper material) can still effectively produce current. In this case light would be absorbed along the longer axial dimension of the semiconductor wires while the resulting electrons or holes would be collected along the much shorter radial dimension in a massively parallel array. Photosensitive junctions employing radial charge-carrier collection can exhibit good energy conversion efficiencies even with relatively low-cost, low-grade bulk materials. Importantly, impure semiconductor materials are much more amenable to scalable, inexpensive manufacturing and processing methods as compared to expensive single crystals. The bulk purity constraints of materials employing a radial charge collection strategy are relaxed due to the orthogonalization of light absorption and charge-carrier collection. As long as the radius of the high aspect ratio rod electrode is no greater than the value of $L$ in the bulk of the semiconductor, photogenerated charges can be collected efficiently (Figure 4).

\subsubsection{Silicon Rod Array: "Solar Carpet"}

In working towards the creation of inexpensively processable radial junction solar cells, highly ordered Si wire arrays grown on a single crystal wafer were transferred into a transparent, flexible polymer matrix. ${ }^{8}$ Arrays of vertically oriented Si wires were grown on Si wafers by photolithographically patterning a catalyst metal onto an oxide buffer layer, followed by vapor-liquid-solid (VLS) growth with $\mathrm{SiCl}_{4}$ gas. The wires were then cast in polydimethylsiloxane (PDMS), a low cost polymer, which was subsequently cured and mechanically separated from the substrate. The resulting wire/polymer composite layers were highly flexible while still maintaining the array alignment and fidelity. Furthermore, the single crystal growth substrate was demonstrated to be reusable by chemically regenerating the patterned oxide template and electrodepositing fresh catalyst metal into it. The scheme showed in Fig. 5 has the potential to enable the economic, roll-to-roll processing of wire array solar cells by incorporating inorganic semiconductor material grown on a recyclable substrate into an inexpensive, flexible organic layer. 


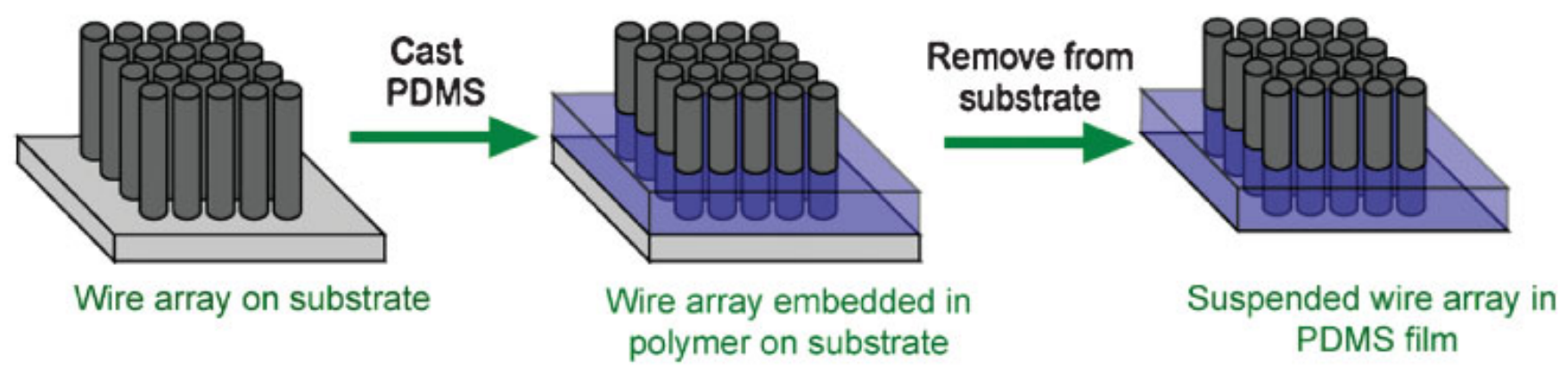

Figure 5. Schematic representation of the wire array embedding and removal process. ${ }^{8}$
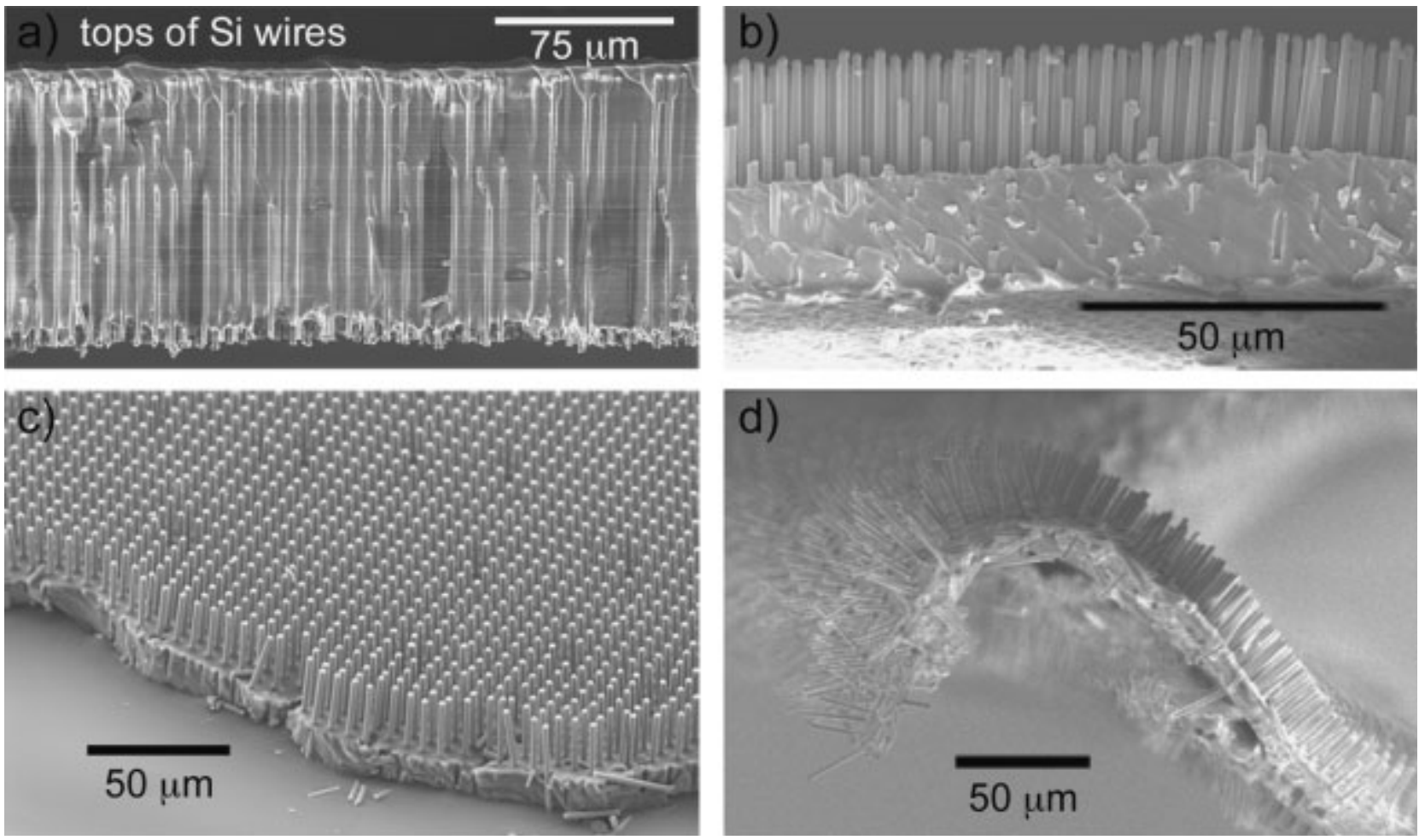

Fig. 6. Cross-sections of embedded Si wire arrays of two different polymer thicknesses. a) Cross-section of a composite film. Upon removal from the growth substrate, wires were exposed only at the bottom of the film. Note the conformal filling of the gaps between wires. b-d) The thinner composite film fabricated had a significant fraction of the wire length emerging from the polymer while the array pattern was maintained. b) The bottom of the film can be seen, demonstrating that the rods passed through the polymer. c) The array order from the top of the film is visible. d) The films remained flexible even while containing ordered arrays of single-crystalline wires. ${ }^{8}$

As can be seen in Figure 6, this massively parallel array resembling carpet fibers on a microscale, for that we called it "solar carpet." Preliminary studies on the current-voltage characteristics of these Si wire array/polymer composite films have been conducted by employing them as photocathode in solar cells. The results have shown that the pattern fidelity and vertical alignment of a rod array can be maintained in a flexible polymer layer. The solar carpet is a step forward toward the employ of Si rod arrays as the photocathode in the three-component solar cell device. 


\subsection{Tungsten oxide as a photoanode material}

Since the pioneering work by Fujishima, demonstrating that crystalline $\mathrm{TiO}_{2}$ could split water to produce $\mathrm{H}_{2}$ and $\mathrm{O}_{2}$ after UV photoexcitation, ${ }^{10}$ wide bandgap metal oxides have been extensively analyzed for water splitting due to their good stability, non-toxicity, low cost and catalytic activity. However, the overall efficiency of the metal oxide is still too low.

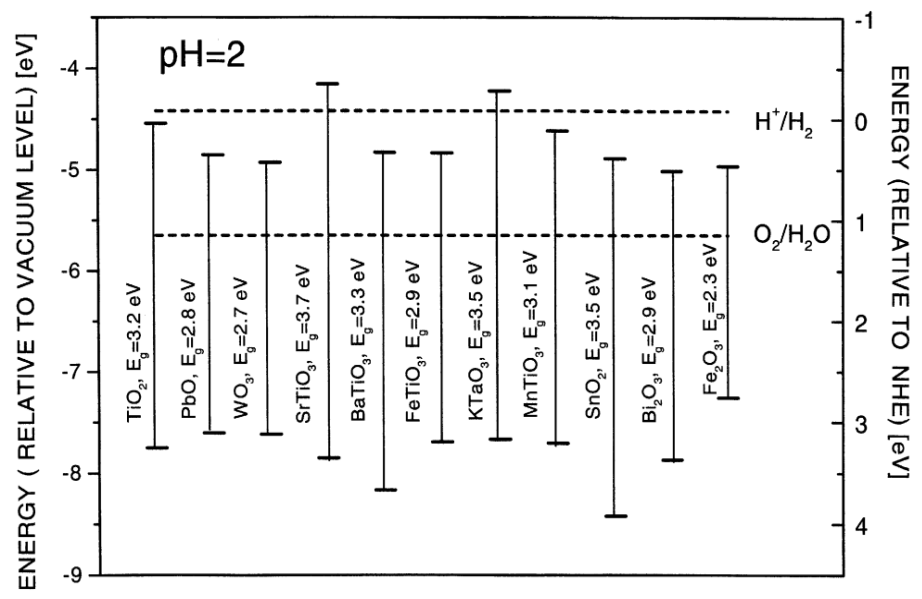

Figure 7. Diagram showing band gap energy of different oxide materials and relative energies with respect in terms of vacuum level and normal hydrogen electrode level in electrolyte of $\mathrm{pH}=2 .^{5}$

Tungsten oxide is a good candidate to drive the water oxidation because it absorbs a reasonable fraction of the solar spectrum. As can been seen in Figure 7, $\mathrm{WO}_{3}$ has a lower bandgap $(2.7 \mathrm{eV})$ than $\mathrm{TiO}_{2}(3.2 \mathrm{eV})$. In addition, $\mathrm{WO}_{3}$ is an earth abundant metal, non-toxic and non-expensive. Since 1976, several photoelectrochemical studies have demonstrated that $\mathrm{WO}_{3}$ could be used as photoanode for water oxidation. ${ }^{11-16}$ However, the efficiency of these $\mathrm{WO}_{3}$ electrodes is still low. One of the most important factors that are limiting the efficiency is the fast electron-hole recombination. With a better charge-carrier collection, the probability that the electron and hole could be used for water splitting before they recombine is higher.

\subsubsection{Porous materials}

For solar cell purpose, porous films have several advantages in comparison with particles and compact systems. In contrast to the random nanoparticle systems where slow electron diffusion typically increases the electron/hole recombination, organized porous structures have excellent electron percolation pathways.

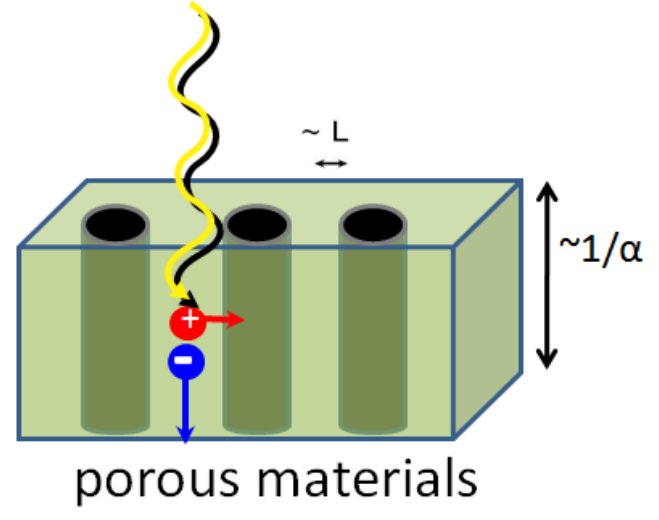

Figure 8. Light absorption pathlength depth $(1 / \alpha)$ and the minority charge-carrier collection length, $L$, in semiconductor photovoltaic or photoelectrochemical junctions in porous materials. 
As rod arrays (Figure 4), porous structures could significantly improve the photocurrent efficiency due to the radial charge-carrier collection (Figure 8). The radial charge-carrier collection of Si porous materials was studied previously in Lewis group. ${ }^{17}$ In addition, porous films have larger area of semiconductor-electrolyte junction than compact layers.

\subsubsection{Porous tungsten oxide film: "Solar Sponge"}

For several decades, porous aluminum oxides have been produced successfully by anodization. Recently, this technique has been expanding to other metals such as $\mathrm{Al}, \mathrm{Ti}$ and $\mathrm{W}$. Several photoelectrochemical studies shows that porous structures have higher photocatalytic capacity than planar films. ${ }^{18,19}$ Currently, Lewis group is working with the optimization of porous $\mathrm{WO}_{3}$ electrodes. Porous $\mathrm{WO}_{3}$ have been produced by potentiostatic anodization of $\mathrm{W}$ foil in $\mathrm{NaF}$ electrolytes (Figure 9). In particular, we are optimizing the pore diameter and thickness of these porous films to allow for efficient carrier collection and solar light absorption.

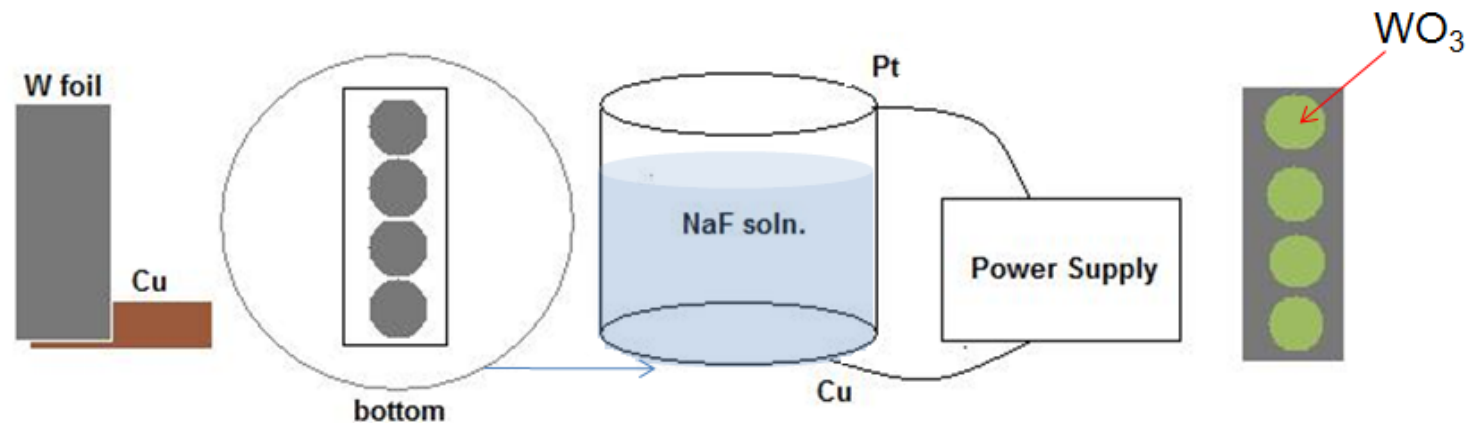

Figure 9. Schematic representation of the $\mathrm{W}$ foil anodization.

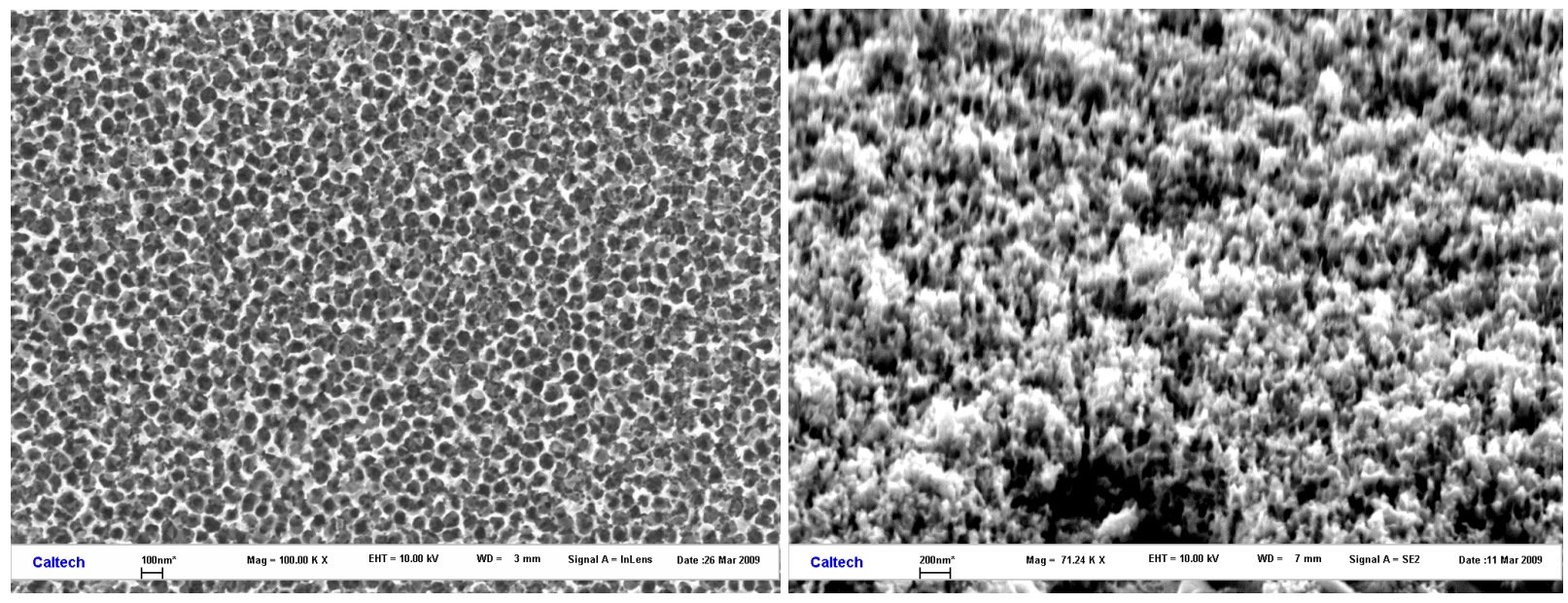

Figure 10.(Right) Top view and (left) tilted view (60 degrees) SEM images of porous $\mathrm{WO}_{3}$ film.

As you can see in Figure 10, very organized porous structures can be produced using this anodization technique. The tilted view shows a very spongy film. This sponge-like structure absorbs light efficiently due to its high surface area; hence we called it "solar sponge". Preliminary studies on the current-voltage characteristics of these porous films have been conducted by employing them as photoanode in solar cells. This solar sponge has a great potential to be used as the photoanode in the three-component solar cell. 


\section{CONCLUSIONS}

A three-component solar water splitting cell has been developed that is composed of only inexpensive, earth-abundant materials, and is simultaneously efficient, durable, and non-expensive. Rod-array and porous electrodes will be employed to enable use of low cost materials and processing conditions by relaxing constraints on the material purity and properties. The semiconductors of this water-splitting device have been synthesized and optimized separately. To date, the most promising materials are p-Si as the photocathode and $\mathrm{WO} 3$ as the photoanode. This work demonstrates the feasibility of materials to approach the production of scalable, photoelectrochemically based solar water-splitting devices for production of hydrogen from the Sun.

\section{ACKNOWLEDGMENTS}

We acknowledge every former and current member of the Lewis group for their contribution to this work. We also acknowledge all members of the Powering the Planet Center for Chemical Innovation (CCI Solar) for their valuable contribution to this project. In addition, we acknowledge the DOE, NSF and BP plc for financial support that has made this work possible. Conference Record

of the IEEE 4th World Conference on Photovoltaic Energy Conversion 1, 221 (2006)

\section{REFERENCES}

[1] Coontz, R.; Hanson, B., "Not so Simple", Science 305, 957 (2004).

[2] Lewis, N. S., "Powering the Planet", MRS Bulletin 32, 809 (2007).

[3] Grätzel, M., "Photoelectrochemical Cells", Nature 2001, 414, 338.

[4] Bolton, J. R., "Solar Fuels", Science 202, 705 (1978).

[5] Bak, T.; Nowotny, J.; Rekas, M.; Sorrell, C. C., "Photo-electrochemical hydrogen generation from water using solar energy. Material-related aspects", Int. J. Hydrogen Energy 27, 991 (2002).

[6] Fahrenbruch, A. L.; Bube, R. H., [Fundamentals of solar cells: Photovoltaic solar energy conversion] Academic Press: New York, 559 (1983).

[7] Kayes, B. M.; Spurgeon, J. M.; Sadler, T. C.; Lewis, N. S.; Atwater, H. A., "Synthesis and Characterization of Silicon Nanorod Arrays for Solar Cell Applications", Conference Record of the IEEE 4th World Conference on Photovoltaic Energy Conversion 1, 221 (2006).

[8] Plass, K. E.; Filler, M. A.; Spurgeon, J. M.; Kayes, B. M.; Maldonando, S.; Brunschwig, B. S.; Atwater, H. A.; Lewis, N. S., "Flexible Polymer-Embedded Si Wire Arrays", Adv. Mater. 21, 325 (2009).

[9] Spurgeon, J. M.; Plass, K. E.; Kayes, B. M.; Brunschwig, B. S.; Atwater, H. A.; Lewis, N. S., "Repeated Epitaxial Growth and Transfer of Arrays of Patterned, Vertically Aligned, Crystalline Si Wires from a Single Si(111) Substrate", Appl. Phys. Lett. 93, 032112 (2008).

[10] Fujishima, A.; Honda, K., "Electrochemical Photolysis of Water at a Semiconductor Electrode", Nature (London) 238, 37 (1972).

[11] Hodes, G.; Cahen, D.; Manassen, J., "Tungsten trioxide as a photoanode for a photoelectrochemical cell (PEC)", Nature 260, 312 (1976).

[12] Miller, E. L.; Marsen, B.; Cole, B.; Lum, M., "Low-Temperature Reactively Sputtered Tungsten Oxide Films for Solar-Powered Water Splitting Applications" Electrochem. Solid-State Lett. 9, (7), G248 (2006).

[13] Santato, C.; Ulmann, M.; Augustynski, J., "Enhanced Visible Light Conversion Efficiency Using NanocrystallineWO ${ }_{3}$ Films", Adv. Mater. 13, (7), 511 (2001).

[14] Santato, C.; Ulmann, M.; Augustynski, J., "Photoelectrochemical Properties of Nanostructured Tungsten Trioxide Films", J. Phys. Chem. B 105, (5), 936 (2001). 
[15] Wang, H.; Lindgren, T.; He, J.; Hagfeldt, A.; Lindquist, S.-E., "Photoelectrochemistry of Nanostructured $\mathrm{WO}_{3}$ Thin Film Electrodes for Water Oxidation: Mechanism of Electron Transport", J. Phys. Chem. B 104, 5686 (2000).

[16] Butler, M. A.; Nasby, R. D.; Quinn, R. K., "Tungsten Trioxide as an Electrode for Photoelectrolysis of Water", Solid State Commun. 19, 1011 (1976).

[17] Maiolo, J. R.; Atwater, H. A.; Lewis, N. S., "Macroporous Silicon as a Model for Silicon Wire Array Solar Cells", J. Phys. Chem. C 112, 6194 (2008).

[18] Berger, s.; Tsuchiya, H.; Ghicov, A.; Scmuki, P., "High photocurrent conversion efficiency in self-organized porous $\mathrm{WO}_{3} "$ ", Applied Physics Letters 88, 203119 (2006).

[19] Guo, Y.; Quan, X.; Lu, N.; Zhao, H.; Chen, S., "High Photocatalytic Capability of Sel-Assembled Nanoporous $\mathrm{WO}_{3}$ with Preferential Orientation of (002) Planes", Environ. Sci. Technol. 41, 4422 (2007). 